L.G.C. Silva

https://orcid.org/0000-0001-5309-0386 T. Casarin

https://orcid.org/0000-0002-4195-0518 L.B. Farias

https://orcid.org/0000-0002-3275-5198

\section{Enrofloxacina de rápida ação associada ao tratamento com eletrólitos orais e reposição energética no tratamento de broncopneumonia neonatal em bezerros Holandeses}

\author{
[Fast action enrofloxacin associated with support treatment with oral \\ electrolyte and energy replacement in the treatment of neonatal \\ bronchopneumonia in Holstein calves]
}

L.G.C. Silva, T. Casarin, L.B. Farias, A.S. Maffi, I.M. Cavazini, U.S. Londero, J. Feijó, F.A.B. Del Pino, V.R. Rabassa, M.N. Corrêa https://orcid.org/0000-0003-2242-9679 I.M. Cavazini U.S. Londero

https://orcid.org/0000-0001-5595-1223 J. Feijó

https://orcid.org/0000-0002-0233-3939 F.A.B.Del Pino

https://orcid.org/0000-0002-5142-5215 V.R. Rabassa

https://orcid.org/0000-0002-0088-0605 M.N. Corrêa

https://orcid.org/0000-0003-0855-2750 https://orcid.org/0000-0003-2498-9725

Universidade Federal de Pelotas - Pelotas, RS

\begin{abstract}
RESUMO
O objetivo deste estudo foi avaliar estratégias terapêuticas para o tratamento de infecções broncopulmonares, utilizando a enrofloxacina de ação rápida e sua associação com suporte e fluidoterapia endovenosa ou suporte e solução oral energética e eletrolítica, por meio da mensuração de parâmetros clínicos, hematológicos, bioquímicos e desenvolvimento corporal de neonatos bovinos. Foram utilizadas 35 bezerras da raça Holandesa, monitoradas desde o nascimento até a sexta semana de vida, divididas aleatoriamente nos grupos: grupo CONTROLE; grupo antibiótico; grupo antibiótico + suporte + fluidoterapia endovenosa; grupo antibiótico + suporte + solução oral; e grupo SUPORTE. Os parâmetros zootécnicos foram avaliados do nascimento até a sexta semana de vida, e os parâmetros hematológicos e bioquímicos foram avaliados zero, 24, 72 e 120 horas após diagnóstico da broncopneumonia. Os animais do grupo antibiótico + suporte + solução oral apresentaram menores níveis de eosinófilos e maiores níveis de neutrófilos segmentados em comparação aos animais dos demais grupos. Não houve diferença nos parâmetros zootécnicos avaliados. Neste estudo, o tratamento com antibiótico e solução oral ofereceu aos animais melhor aporte para combater a broncopneumonia, favorecendo o organismo a desenvolver uma resposta imune efetiva diante da infecção.
\end{abstract}

Palavras-chave: bezerros, doença respiratória, parâmetros hematológicos, fluidoterapia

\begin{abstract}
The objective of this study was to evaluate therapeutic strategies for treatment of bronchopulmonary infections using fast-acting enrofloxacin and its association with support and endovenous fluid or support and oral energy and electrolytic solution, by measuring clinical, hematological, biochemical and development parameters of bovine neonates. Thirty-five Holstein calves, monitored from birth to six weeks of age, were randomly divided into five groups: control group; antibiotic group; antibiotic group + support + intravenous fluid therapy; antibiotic group + support + oral solution; and support group. The performance parameters were evaluated from birth to the $6^{\text {th }}$ week of age and hematological and biochemical parameters were evaluated 0, 24, 72 and 120 hours after diagnosis of bronchopneumonia. Calves of the antibiotic group + support + oral solution group presented lower levels of eosinophils and higher levels of segmented neutrophils compared to the other groups. There was no difference in performance parameters evaluated. In this study, the treatment with antibiotic and oral solution offered the animals had a better contribution to treat bronchopneumonia, favoring the organism to develop an effective immune response to that infection.
\end{abstract}

Keywords: calves, respiratory disease, haematological parameters, fluid therapy

\section{INTRODUÇÃO}

Em rebanhos leiteiros, problemas na criação das bezerras podem afetar diretamente a eficiência produtiva, devido à diminuição no ganho de peso

Recebido em 10 de julho de 2018

Aceito em 29 de abril de 2019

E-mail: lucasbalinhas@gmail.com e ao atraso da puberdade, além de comumente afetar o início da produção leiteira e, em alguns casos, levar o animal ao óbito (Virtala et al., 1996; Stanton et al., 2012; Closs e Dechow, 2017). Nas primeiras semanas de vida, há uma alta incidência de doenças em bezerras, muitas 
vezes causadas por erros de manejo, como na transferência de imunidade passiva colostral, ou por ambiente altamente infectado (Langel et al., 2015). Os distúrbios respiratórios, como a broncopneumonia, são a segunda maior causa de morte nessa categoria, sendo superada apenas pela diarreia neonatal (Fratric et al., 2012; Langel et al., 2015; Buac et al., 2016; Buczinski et al., 2016).

A broncopneumonia é causada por diversos fatores decorrentes da interação entre agentes infecciosos e não infecciosos. Entre os agentes infecciosos, há os vírus herpes vírus bovino tipo1 (BHV-1), parainfluenza tipo 3 (PI-3V), diarreia viral bovina (BVDV) e o vírus respiratório sincicial bovino (BRSV), as bactérias Mannheimia haemolytica, Pasteurella multocida, Haemophilus somnus, Chlamydophila psittaci, Chlamydophila pecorum, Arcanobacterium pyogenes e o Mycoplasma bovis. Referente aos fatores não infecciosos, há principalmente o manejo, visto que fatores de estresse ambiental diminuem a defesa imune (Fratric et al., 2012; Buac et al., 2016).

Os quadros de broncopneumonia geram elevadas perdas econômicas pelo custo do tratamento, além do atraso no crescimento e de óbitos dos neonatos (Gonçalves et al., 2001; Heins et al., 2014). Apesar da constante busca por alternativas terapêuticas antimicrobianas eficazes no tratamento da broncopneumonia (Davis et al., 2007; Heins et al., 2014), ainda não foi encontrada uma estratégia medicamentosa que reduza significativamente a taxa de mortalidade desses animais. Como alternativa, uma nova formulação de antibiótico do grupo das quinolonas - a enrofloxacino com rápida ação foi lançada nos últimos anos. Esse medicamento atinge rapidamente concentrações terapêuticas na circulação e nos pulmões em 30 e 60 minutos, respectivamente, e seu pico plasmático é alcançado entre cinco e seis horas, proporcionando rápida resposta terapêutica após aplicação de dose única do produto (Kaartinen et al., 1995; Montagner et al., 2014).

Grande parte dos tratamentos preconiza somente o uso de antibióticos, sem considerar os distúrbios hidroeletrolíticos e a deficiência energética, ocasionados pela anorexia durante a doença (Radostits et al., 2002), o que pode tornar muitas vezes o tratamento ineficiente, causando um retardo no desenvolvimento corporal (Graham et al., 2010). Em bezerros, o tratamento de eleição para correção de distúrbios hidroeletrolíticos é a infusão intravenosa contínua de solução isotônica (Radostits et al., 2002), no entanto essa abordagem terapêutica demanda tempo para que o volume necessário seja administrado, sendo muitas vezes realizado de forma errônea. Diante disso, a administração oral de repositores eletrolíticos e energéticos surge como uma opção viável e eficaz (Walker $e t$ al., 1998), podendo restabelecer o equilíbrio eletrolítico, reidratar o animal e favorecer o restabelecimento do apetite e a resposta clínica ao tratamento antimicrobiano.

Diante do exposto, o objetivo deste estudo foi avaliar estratégias terapêuticas para o tratamento de infecções broncopulmonares, utilizando a enrofloxacina de ação rápida e sua associação com suporte e fluidoterapia endovenosa ou suporte e solução oral eletrolítica e energética, por meio da mensuração de parâmetros clínicos, hematológicos, bioquímicos e desenvolvimento corporal de neonatos bovinos.

\section{MATERIAL E MÉTODOS}

Todos os procedimentos foram aprovados pelo Comitê de Ética em Experimentação Animal da Universidade Federal de Pelotas (UFPel) (código CEEA: 2827). Este estudo foi realizado em uma fazenda comercial, localizada no sul do Rio Grande do Sul, RS (32,8 $8^{\circ} 16^{\prime}$ S, 52, $\left.8^{\circ} 32^{\prime} \mathrm{L}\right)$. Foram utilizadas 35 bezerras da raça Holandesa, monitoradas desde o nascimento até a sexta semana de vida. Após o parto, os animais permaneceram aproximadamente $12 \mathrm{~h}$ com as vacas, a fim de receberem colostro naturalmente, de acordo com o manejo da fazenda, sendo posteriormente alojados em sistema de criação individual. A dieta fornecida aos animais era composta de quatro litros de leite por dia, divididos em duas refeições (sete e 17 horas), com acesso livre ao concentrado peletizado (Supra Terneira Laminado, Supra ${ }^{\circledR}$, São Leopoldo, Brasil), de acordo com o NRC (2001), e água ad libitum.

Durante o período experimental, foram realizados exames clínicos, duas vezes por semana, em todos os animais. As funções vitais avaliadas foram frequência cardíaca (FC) e respiratória (FR), coloração de mucosas, 
temperatura corporal e tempo de perfusão capilar (TPC), por meio dos parâmetros propostos por Raiser (2003). Além disso, foi observado diariamente o comportamento delas, quanto ao grau de consciência e apetite. Ao ser observada alteração desses parâmetros, realizava-se o exame clínico específico do sistema respiratório.

$\mathrm{Na}$ avaliação clínica específica do aparelho respiratório, foram monitorados o tipo respiratório e a presença de secreção nasal, bem como foi realizada a auscultação da área de projeção pulmonar no tórax para determinação da presença de estertores crepitantes (Gonçalves et al., 2001). Quando identificada a broncopneumonia (presença de febre e estertoração pulmonar), os animais foram avaliados nos momentos zero, 24, 72 e $120 \mathrm{~h}$ em relação ao diagnóstico. Semanalmente (desde o nascimento até a sexta semana de vida), foram realizadas mensurações de altura da cernelha, largura da garupa e perímetro torácico. Nos mesmos dias, os animais eram pesados, com uso de fita de pesagem indicada para bovinos, sempre antes do fornecimento da dieta matutina. A partir dos dados de peso, foi determinado o ganho médio diário (GMD).

Os animais diagnosticados com broncopneumonia foram aleatoriamente divididos em quatro tratamentos experimentais: grupo antibiótico (ATB; n=9), que recebeu a aplicação de enrofloxacina de rápida ação (Kinetomax ${ }^{\circledR}$, Bayer Saúde Animal, Alemanha), em dose única de $7,5 \mathrm{mg} / \mathrm{kg}$ de peso vivo (PV), por via intramuscular (IM), no momento do diagnóstico da doença; grupo antibiótico + suporte + fluido endovenoso (ATB+S+FEV+; $\mathrm{n}=4)$, que recebeu a aplicação de enrofloxacina de rápida ação, igual ao grupo ATB; mais a administração de cloridrato de bromexina (Aliv $\mathrm{V}^{\circledR}$, Agener União, Brasil), em dose diária de $0,3 \mathrm{mg} / \mathrm{kg}$ de PV, por três dias, via IM; também flunixina meglumina (Flunamine ${ }^{\circledR}$, Bayer Saúde Animal, Alemanha), em dose de $1,1 \mathrm{mg} / \mathrm{kg}$ de $\mathrm{PV}$, por via IM, por três dias; e fluidoterapia endovenosa (EV) com $\mathrm{NaCl} 0,9 \%$, em volume estabelecido de acordo com o grau de desidratação (Langel et al.); grupo antibiótico + suporte + solução oral $(\mathrm{ATB}+\mathrm{S}+\mathrm{SO} ; \mathrm{n}=8)$, que recebeu a aplicação do mesmo tratamento do grupo ATB $+\mathrm{S}+\mathrm{FEV}$, apenas trocando o fluido endovenoso por uma suplementação com solução de reposição oral composta por acetato de sódio, glicose, cloreto de sódio e cloreto de potássio (Glutellac $^{\circledR}$, Bayer Saúde Animal, Alemanha), na dose de $50 \mathrm{~mL}$, durante três dias; e o grupo SUPORTE (SUPORTE; $\mathrm{n}=8$ ), que recebeu a administração de cloridrato de bromexina (Aliv $\mathrm{V}^{\circledR}$, Agener União, Brasil), em dose diária de $0,3 \mathrm{mg} / \mathrm{kg}$ de PV, por três dias, via IM; flunixina meglumina (Flunamine ${ }^{\circledR}$, Bayer Saúde Animal, Alemanha), em dose de $1,1 \mathrm{mg} / \mathrm{kg}$ de $\mathrm{PV}$, por três dias; e fluidoterapia $\mathrm{EV}$ à base de $\mathrm{NaCl} 0,9 \%$, em volume a ser estabelecido de acordo com o grau de desidratação. O grupo CONTROLE foi composto pelos animais que não apresentaram a enfermidade, animais sadios (CONTROLE; $\mathrm{n}=5$ ).

Amostras de sangue $(15 \mathrm{~mL})$ foram coletadas dos animais doentes nos momentos zero, 24, 72 e 120h, em relação ao diagnóstico, enquanto animais sadios foram coletados duas vezes por semana, por meio da venopunção jugular. O hemograma foi realizado pela contagem de hemácias e leucócitos totais, em contador automático CC-530 ${ }^{\circledR}$ (CELM, São Paulo, Brasil). Já o teor plasmático de fibrinogênio foi obtido pelo método de precipitação pelo calor e leitura em refratômetro (Millar et al., 1971). A contagem diferencial de leucócitos e a estimativa do número total de plaquetas foram realizadas por meio de esfregaço sanguíneo corado com corante de panótipo rápido, em microscopia óptica (Thrall, 2007). E o volume globular foi obtido por microcapilares de $50 \mu \mathrm{L}$ submetidos à centrifugação a 14.000 x g, durante cinco minutos, e, em seguida, foi realizada leitura em cartão especial para volume globular.

Nas análises bioquímicas, para se determinarem as concentrações de proteína plasmática total (PPT), albumina e glicose, utilizaram-se reagentes comerciais (Labtest ${ }^{\circledR}$, Belo Horizonte, Brasil), manuseados conforme recomendação do fabricante. A leitura das amostras foi realizada por meio de espectrofotômetro de luz visível BioEspectro SP $220^{\circledR}$ (Bioespectro, Curitiba, Brasil). O nível de globulina foi definido pela diferença entre os valores de PPT e albumina. E o índice albumina:globulina $(\mathrm{A}: \mathrm{G})$ foi calculado dividindo-se o valor da fração albumina pelo valor total da fração globulina de cada amostra analisada. A relação proteína plasmática total com fibrinogênio (PPT:F) foi obtida diminuindose a concentração de proteína plasmática pela concentração de fibrinogênio, dividindo-se essa 
resultante pela concentração de fibrinogênio (Jain, 1993). Em todas as análises, o coeficiente de variação foi inferior a $10 \%$.

O lavado traqueobrônquico foi realizado no dia do diagnóstico da broncopneumonia, por meio de traqueocentese, como descrito por Gonçalves et al. (1991). O material coletado foi armazenado em recipiente estéril e armazenado a $-20^{\circ} \mathrm{C}$, até a realização de isolamento bacteriano e teste de sensibilidade ao antibiótico enrofloxacino de rápida ação, no Laboratório Regional de Diagnóstico da UFPel.

Os dados foram analisados no programa SAS (SAS Institute Inc., Cary, EUA). Os resultados das variáveis FC, FR, temperatura, TPC, contagem de hemácias, leucócitos totais, neutrófilos segmentados, neutrófilos bastonetes, linfócitos, monócitos, eosinófilos, fibrinogênio, PPT, albumina, globulina, PPT:F, A:G, glicose, peso, altura de cernelha, largura da garupa, perímetro torácico e GMD foram submetidos a testes de normalidade de Shapiro-Wilk. Depois, as médias foram analisadas pelo método MIXED MODELS, considerando-se o animal, o grupo, a hora e suas interações. A comparação de médias foi feita pelo teste de Tukey-Kramer. As variáveis de tipo respiratório, presença de estertoração pulmonar e secreção nasal foram observadas categoricamente e analisadas pelo qui-quadrado. Foram considerados significativos os valores de $\mathrm{P}<0,05 \mathrm{e}$, como tendência estatística, os valores de $\mathrm{P}<0,10$. Os resultados de isolamento bacteriano e antibiograma foram analisados somente de forma descritiva.

\section{RESULTADOS}

Pelo exame bacteriológico das 30 amostras de lavado traqueobrônquico, somente oito amostras apresentaram crescimento bacteriano, e as bactérias isoladas foram: Bacillus spp $(\mathrm{n}=5$, 62,5\%); Pasteurella multocida ( $\mathrm{n}=1,12,5 \%)$; Staphylococos Gram+ $(\mathrm{n}=1, \quad 12,5 \%) ; \quad \mathrm{e}$ Arcanobacterium pyogenes $(\mathrm{n}=1,12,5 \%)$. Das bactérias isoladas, a Pasteurella multocida foi resistente à enrofloxacina de rápida ação, enquanto as demais bactérias foram sensíveis ao antibiótico.
No exame clínico específico, observou-se maior porcentagem de animais apresentando estertoração pulmonar nas $72 \mathrm{~h}$ pós-diagnóstico no grupo SUPORTE, em comparação com os demais grupos $(\mathrm{P}<0,05)$. Em relação ao grau de consciência, à coloração de mucosas, à secreção nasal e ao tipo respiratório, os animais não apresentaram diferenças entre os grupos quanto a essas observações em $72 \mathrm{~h}$ pós-diagnóstico ( $\mathrm{P}>0,05)$. No exame clínico geral, foi observada alteração apenas na $\mathrm{FC}$, tendo o grupo ATB $+\mathrm{S}+\mathrm{SO}$ obtido aumento da frequência cardíaca em relação aos grupos ATB, ATB+S+FEV e SUPORTE, porém não diferindo do grupo CONTROLE (Tab. 1). Os demais parâmetros, FR, temperatura e TPC, foram semelhantes entre os grupos ( $\mathrm{P}>0,05$; Tab. 1). Em relação aos parâmetros hematológicos, os grupos ATB, ATB+S+FEV e SUPORTE apresentaram menor número de hemácias em relação ao grupo CONTROLE, não havendo diferença entre o grupo CONTROLE e $\mathrm{ATB}+\mathrm{S}+\mathrm{SO}$ (Tab. 1). Quanto à contagem de leucócitos totais, o grupo SUPORTE apresentou maior número que os grupos CONTROLE e $\mathrm{ATB}+\mathrm{S}+\mathrm{SO}$.

No diferencial leucocitário, foram observadas diferenças nas quantidades de neutrófilos segmentados, neutrófilos bastonetes, linfócitos, eosinófilos e monócitos. Em relação aos níveis de linfócitos, o grupo SUPORTE apresentou valores mais elevados, que diferiram dos grupos CONTROLE, ATB+S+FEV e ATB+S+SO $(\mathrm{P}=0,02)$, não diferindo do grupo ATB $(\mathrm{P}>0,05)$. Além disso, os animais do grupo ATB tiveram maiores níveis de linfócitos em comparação ao grupo $\mathrm{ATB}+\mathrm{S}+\mathrm{SO}(\mathrm{P}=0,02 ; \mathrm{Tab}$. 1). Os níveis de neutrófilos segmentados foram superiores no grupo $\mathrm{ATB}+\mathrm{S}+\mathrm{SO}$, em comparação aos demais grupos $(\mathrm{P}<0,01)$, havendo uma interação grupo*hora $(\mathrm{P}=0,03)$. Os animais do grupo $\mathrm{ATB}+\mathrm{S}+\mathrm{SO}$ apresentaram níveis mais elevados de neutrófilos segmentados 24 e 72 horas após o diagnóstico de broncopneumonia (Fig. 1). Já os níveis de eosinófilos foram inferiores no grupo $\mathrm{ATB}+\mathrm{S}+\mathrm{SO}$ em relação aos demais grupos $(\mathrm{P}=0,03$; Tab. 1). A concentração de monócitos, bastonetes e hemoglobina não apresentou diferenças significativas entre os grupos $(\mathrm{P}>0,05$; Tab. 1). 
Enrofloxacina de rápida...

Tabela 1. Médias e erro médio-padrão (EMP) dos parâmetros clínicos, hematológicos e bioquímicos de bezerras submetidas a diferentes tratamentos para broncopneumonia neonatal bovina

\begin{tabular}{|c|c|c|c|c|c|c|c|c|}
\hline Parâmetros & Controle & ATB & $\mathrm{ATB}+\mathrm{S}+\mathrm{FEV}$ & Suporte & $\mathrm{ATB}+\mathrm{S}+\mathrm{SO}$ & Grupo & Hora & Grupo*Hora \\
\hline \multicolumn{9}{|l|}{ Clínicos } \\
\hline $\begin{array}{l}\mathrm{FC} \\
(\mathrm{bpm})\end{array}$ & $\begin{array}{l}114.85^{\mathrm{ab}} \pm \\
6,00\end{array}$ & $\begin{array}{l}103.69^{\mathrm{b}} \pm \\
4,47\end{array}$ & $\begin{array}{l}103 \cdot 25^{\mathrm{b}} \pm \\
6,71\end{array}$ & $\begin{array}{l}103.94^{\mathrm{b}} \pm \\
4,75\end{array}$ & $\begin{array}{l}125 \cdot 39^{\mathrm{a}} \pm \\
4,47\end{array}$ & 0,03 & 0,39 & 0,91 \\
\hline $\begin{array}{l}\text { FR } \\
(\mathrm{mpm})\end{array}$ & $\begin{array}{l}68.85 \pm \\
6,70\end{array}$ & $\begin{array}{l}73.72 \pm \\
4,88\end{array}$ & $\begin{array}{l}84.00 \pm \\
7,32\end{array}$ & $\begin{array}{l}75.68 \pm \\
5,26\end{array}$ & $\begin{array}{l}71.07 \pm \\
5,03\end{array}$ & 0,57 & 0,83 & 0,37 \\
\hline $\begin{array}{l}\text { Temperatura } \\
\left({ }^{\circ} \mathrm{C}\right)\end{array}$ & $\begin{array}{l}39,23 \pm \\
0,2\end{array}$ & $\begin{array}{l}39,65 \pm \\
0,15\end{array}$ & $\begin{array}{l}39,39 \pm \\
0,26\end{array}$ & $\begin{array}{l}39,50 \pm \\
0,15\end{array}$ & $\begin{array}{l}38,60 \pm \\
0,16\end{array}$ & 0,51 & 0,01 & 0,79 \\
\hline $\begin{array}{l}\text { TPC } \\
(\mathrm{seg})\end{array}$ & $\begin{array}{l}1,70 \pm \\
0,18\end{array}$ & $\begin{array}{l}1,86 \pm \\
0,13\end{array}$ & $\begin{array}{l}2,08 \pm \\
0,23\end{array}$ & $\begin{array}{l}2,00 \pm \\
0,14\end{array}$ & $\begin{array}{l}2,00 \pm \\
0,13\end{array}$ & 0,57 & 0,67 & 0,97 \\
\hline \multicolumn{9}{|l|}{ Hematológicos } \\
\hline $\begin{array}{l}\text { Hemácias } \\
\left(10^{6} / \mu \mathrm{L}\right)\end{array}$ & $\begin{array}{l}7,51^{\mathrm{a}} \pm \\
0,24\end{array}$ & $\begin{array}{l}6,93^{\mathrm{bc}} \pm \\
0,18\end{array}$ & $\begin{array}{l}6,42^{\mathrm{c}} \pm \\
0,29\end{array}$ & $\begin{array}{l}6,70^{\mathrm{bc}} \pm \\
0,20\end{array}$ & $\begin{array}{l}7,39^{\mathrm{ab}} \pm \\
0,19\end{array}$ & $<0,01$ & 0,97 & 0,70 \\
\hline $\begin{array}{l}\text { Leucócitos totais } \\
\left(10^{6} / \mu \mathrm{L}\right)\end{array}$ & $\begin{array}{l}9245^{\mathrm{b}} \pm \\
1381,99\end{array}$ & $\begin{array}{l}11811^{\mathrm{ab}} \pm \\
1061,77\end{array}$ & $\begin{array}{l}11650^{\mathrm{ab}} \pm \\
1668,91\end{array}$ & $\begin{array}{l}14746^{\mathrm{a}} \pm \\
1155,76\end{array}$ & $\begin{array}{l}10564^{\mathrm{b}} \pm \\
1122,49\end{array}$ & 0,03 & 0,19 & 0,91 \\
\hline $\begin{array}{l}\text { Hemoglobina } \\
(\mathrm{g} / \mathrm{dL})\end{array}$ & $\begin{array}{l}8,01 \pm \\
0,3\end{array}$ & $\begin{array}{l}8,01 \pm \\
0,22\end{array}$ & $\begin{array}{l}7,28 \pm \\
0,4\end{array}$ & $\begin{array}{l}7,54 \pm \\
0,22\end{array}$ & $\begin{array}{l}7,47 \pm \\
0,45\end{array}$ & 0,32 & 0,93 & 0,99 \\
\hline $\begin{array}{l}\text { Neutrófilos } \\
\text { Segmentados }(/ \mu \mathrm{L})\end{array}$ & $\begin{array}{l}2449,81^{\mathrm{b}} \pm \\
643,97\end{array}$ & $\begin{array}{l}3192,26^{\mathrm{b}} \pm \\
450,82\end{array}$ & $\begin{array}{l}2130,92^{\mathrm{b}} \pm \\
788,70\end{array}$ & $\begin{array}{l}3854,72^{\mathrm{b}} \pm \\
444,38\end{array}$ & $\begin{array}{l}8585,50^{\mathrm{a}} \pm \\
1115,39\end{array}$ & $<0,01$ & 0,25 & 0,03 \\
\hline $\begin{array}{l}\text { Neutrófilos } \\
\text { Bastonetes }(/ \mu \mathrm{L})\end{array}$ & $\begin{array}{l}163,06 \pm \\
47,18\end{array}$ & $\begin{array}{l}215,44 \pm \\
33,49\end{array}$ & $\begin{array}{l}135,46 \pm \\
57,78\end{array}$ & $\begin{array}{l}198,73 \pm \\
38,28\end{array}$ & $\begin{array}{l}280,75 \pm \\
81,72\end{array}$ & 0,56 & 0,40 & 0,20 \\
\hline $\begin{array}{l}\text { Linfócitos } \\
(/ \mu \mathrm{L})\end{array}$ & $\begin{array}{l}6108,56^{\mathrm{bc}} \pm \\
927,08\end{array}$ & $\begin{array}{l}7626,66^{\mathrm{ab}} \pm \\
649,01\end{array}$ & $\begin{array}{l}6377,79^{\mathrm{bc}} \pm \\
1135,43\end{array}$ & $\begin{array}{l}8866,17^{\mathrm{a}} \pm \\
639,74\end{array}$ & $\begin{array}{l}4181,75^{\mathrm{c}} \pm \\
1605,75\end{array}$ & 0,02 & 0,23 & 0,99 \\
\hline $\begin{array}{l}\text { Monócitos } \\
(/ \mu \mathrm{L})\end{array}$ & $\begin{array}{l}285,75 \pm \\
61,81\end{array}$ & $\begin{array}{l}444,09 \pm \\
43,27\end{array}$ & $\begin{array}{l}328,96 \pm \\
75,70\end{array}$ & $\begin{array}{l}461,77 \pm \\
42,65\end{array}$ & $\begin{array}{l}244,50 \pm \\
107,07\end{array}$ & 0,08 & 0,44 & 0,66 \\
\hline $\begin{array}{l}\text { Eosinófilos } \\
(/ \mu \mathrm{L})\end{array}$ & $\begin{array}{l}242,81^{\mathrm{a}} \pm \\
42,60\end{array}$ & $\begin{array}{l}323,11^{\mathrm{a}} \pm \\
33,32\end{array}$ & $\begin{array}{l}260,21^{\mathrm{a}} \pm \\
58,30\end{array}$ & $\begin{array}{l}258,30^{\mathrm{a}} \pm \\
32,84\end{array}$ & $\begin{array}{l}76,50^{\mathrm{b}} \pm \\
60,4\end{array}$ & 0,03 & 0,21 & 0,48 \\
\hline Bioquímicos & & & & & & & & \\
\hline $\begin{array}{l}\text { Fibrinogênio } \\
(\mathrm{g} / \mathrm{dL})\end{array}$ & $\begin{array}{l}0,45 \pm \\
61,34\end{array}$ & $\begin{array}{l}0,559 \pm \\
56,05\end{array}$ & $\begin{array}{l}0,495 \pm \\
8163\end{array}$ & $\begin{array}{l}0,634 \pm \\
61,71\end{array}$ & $\begin{array}{l}0,433 \pm \\
49,82\end{array}$ & 0,09 & 0,17 & 0,80 \\
\hline $\begin{array}{l}\text { PPT } \\
(\mathrm{g} / \mathrm{dL})\end{array}$ & $\begin{array}{l}6,19 \pm \\
0,20\end{array}$ & $\begin{array}{l}6,04 \pm \\
0,15\end{array}$ & $\begin{array}{l}6,58 \pm \\
0,22\end{array}$ & $\begin{array}{l}5,96 \pm \\
0,16\end{array}$ & $\begin{array}{l}5,97 \pm \\
0,15\end{array}$ & 0,18 & 0,64 & 0,99 \\
\hline PPT:fibrinogênio & $\begin{array}{l}18.66 \pm \\
2,28^{\mathrm{ab}}\end{array}$ & $\begin{array}{l}11.53 \pm \\
2,08^{\mathrm{b}}\end{array}$ & $\begin{array}{l}14,83 \pm \\
3,04^{\text {ab }}\end{array}$ & $\begin{array}{l}12,54 \pm \\
2,29^{\mathrm{b}}\end{array}$ & $\begin{array}{l}19,70 \pm \\
1,85^{\mathrm{a}}\end{array}$ & 0,02 & 0,11 & 0,89 \\
\hline $\begin{array}{l}\text { Albumina } \\
(\mathrm{g} / \mathrm{dL})\end{array}$ & $\begin{array}{l}2.36 \pm \\
0,08\end{array}$ & $\begin{array}{l}2.43 \pm \\
0,06\end{array}$ & $\begin{array}{l}2.57 \pm \\
0,10\end{array}$ & $\begin{array}{l}2.33 \pm \\
0,06\end{array}$ & $\begin{array}{l}2.49 \pm \\
0,07\end{array}$ & 0,26 & 0,20 & 0,37 \\
\hline $\begin{array}{l}\text { Globulina } \\
(\mathrm{g} / \mathrm{dL})\end{array}$ & $\begin{array}{l}3.83 \pm \\
0,18\end{array}$ & $\begin{array}{l}3.60 \pm \\
0,13\end{array}$ & $\begin{array}{l}4.01 \pm \\
0,20\end{array}$ & $\begin{array}{l}3.63 \pm \\
0,14\end{array}$ & $\begin{array}{l}3.52 \pm \\
0,13\end{array}$ & 0,30 & 0,93 & 0,99 \\
\hline $\begin{array}{l}\text { Glicose } \\
(\mathrm{mg} / \mathrm{dL})\end{array}$ & $\begin{array}{l}89.69 \pm \\
5,13\end{array}$ & $\begin{array}{l}86.38 \pm \\
3,62\end{array}$ & $\begin{array}{l}77.52 \pm \\
5,58\end{array}$ & $\begin{array}{l}78.81 \pm \\
4,17\end{array}$ & $\begin{array}{l}78.81 \pm \\
3,60\end{array}$ & 0,23 & 0,03 & 0,39 \\
\hline Albumina:globulina & $\begin{array}{l}0,65 \pm \\
0,04\end{array}$ & $\begin{array}{l}0,69 \pm \\
0,03\end{array}$ & $\begin{array}{l}0,65 \pm \\
0,05\end{array}$ & $\begin{array}{l}0,69 \pm \\
0,03\end{array}$ & $\begin{array}{l}0,74 \pm \\
0,03\end{array}$ & 0,42 & 0,55 & 0,65 \\
\hline
\end{tabular}

Grupos: CONTROLE = animais que não apresentaram a doença; ATB = antibiótico; ATB+S+SO = antibiótico + suporte+ solução oral; ATB+S+FEV = antibiótico + suporte + fluido endovenoso; SUPORTE = suporte + fluido endovenoso. Média \pm erro médio-padrão. Parâmetros: FC = frequência cardíaca; FR = frequência respiratória; TPC: tempo de perfusão capilar; PPT = proteínas plasmáticas totais. ${ }^{\text {abc }}$ Médias seguidas de letras minúsculas diferentes no mesmo parâmetro diferem entre grupos pelo teste de Tukey $(\mathrm{P}<0,05)$. 


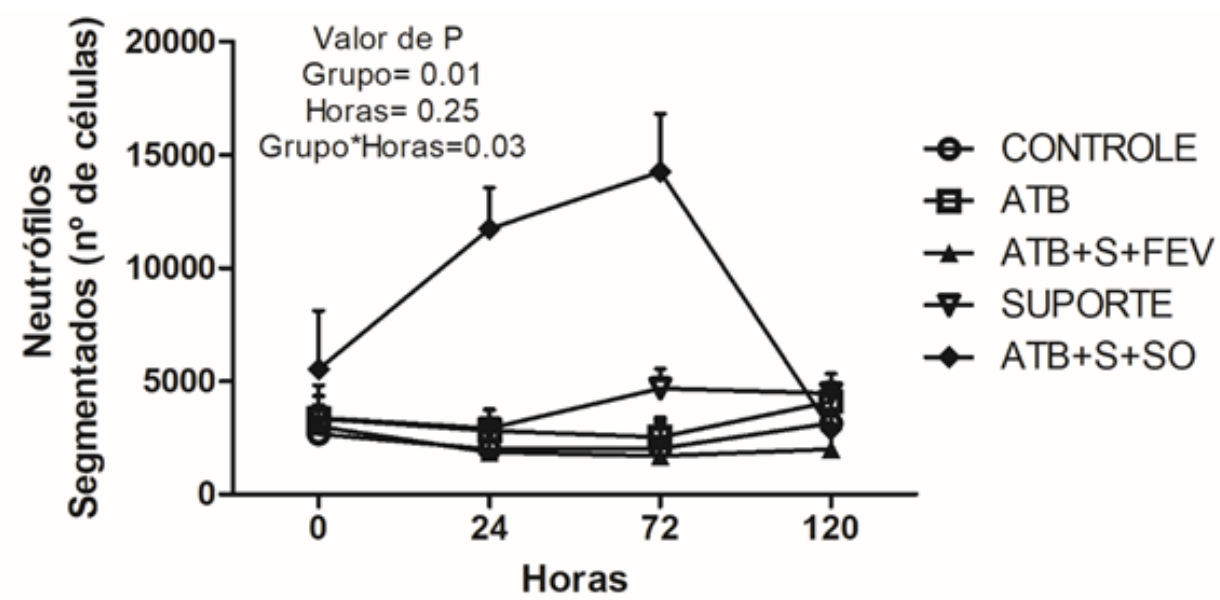

Figura 1. Número de neutrófilos segmentados ( ${ }^{\circ}$ de células) nas horas zero, 24, 72 e 120 após diagnóstico de broncopneumonia de bezerras submetidas a diferentes tratamentos para broncopneumonia neonatal bovina.

Quanto aos marcadores bioquímicos, não houve diferença quanto aos níveis de PPT, albumina, fibrinogênio, globulinas e relação albumina:globulina (A:G) (P>0,05; Tab. 1), porém, para o fibrinogênio, observou-se uma tendência $(\mathrm{P}=0,09)$, apresentando o grupo SUPORTE a maior média. A relação proteínas plasmáticas totais:fibrinogênio (PPT:F) apresentou diferenças entre os grupos $(\mathrm{P}=0,02$; Tab. 1): os grupos $\mathrm{ATB}+\mathrm{S}+\mathrm{SO}$ e CONTROLE demonstraram maior relação do que os grupos SUPORTE e ATB. Entretanto, os grupos
ATB+S+FEV, SUPORTE e ATB não foram diferentes ( $\mathrm{P}>0,05$; Tab. 1). Os níveis de glicose apresentaram diferenças apenas entre as horas de coleta, tendo os animais apresentado menores níveis na hora zero do que nas demais horas (24, 72 e 120h) $(\mathrm{P}=0,03$; Tab. 1).

Em relação aos parâmetros zootécnicos altura de cernelha, largura da garupa, perímetro torácico e ganho de peso, não houve diferença entre os grupos $(\mathrm{P}>0,05)$, como demonstrado na Tab. 2 . Durante este estudo, nenhum animal foi a óbito.

Tabela 2. Médias e erro-padrão médio dos parâmetros zootécnicos de bezerras submetidas a diferentes tratamentos para broncopneumonia, durante as seis primeiras semanas de vida

\begin{tabular}{llllllll}
\hline \multirow{2}{*}{ Parâmetros } & Controle & ATB & ATB+S+FEV & Suporte & ATB+S+SO & P & \\
\cline { 2 - 7 } & Média \pm Erro & Média \pm Erro & Média \pm Erro & Média \pm Erro & Média \pm Erro & Grupo & $\begin{array}{l}\text { Grupo* } \\
\text { Semana }\end{array}$ \\
\hline Peso (kg) & $45,6 \pm 1,80$ & $46,5 \pm 0,69$ & $45,0 \pm 1,19$ & $45,20 \pm 0,94$ & $45,28 \pm 1,82$ & 0,590 & 0,760 \\
GMD (g) & $0,4 \pm 0,09$ & $0,4 \pm 0,04$ & $0,39 \pm 0,07$ & $0,38 \pm 0,39$ & $0,39 \pm 0,08$ & 0,476 & 0,546 \\
Perímetro (cm) & $79,6 \pm 1,34$ & $78,8 \pm 0,56$ & $77,98 \pm 1,03$ & $78,17 \pm 0,82$ & $78,28 \pm 0,76$ & 0,676 & 0,796 \\
Altura (cm) & $81,7 \pm 1,53$ & $81,0 \pm 0,64$ & $81,98 \pm 0,87$ & $81,59 \pm 0,77$ & $81,57 \pm 0,73$ & 0,399 & 0,493 \\
Largura (cm) & $29,1 \pm 0,89$ & $28,7 \pm 0,33$ & $29,11 \pm 0,48$ & $29,12 \pm 0,71$ & $30,06 \pm 0,79$ & 0,401 & 0,219 \\
\hline
\end{tabular}

Grupos: CONTROLE = sadias; $\mathrm{ATB}=$ antibiótico; $\mathrm{ATB}+\mathrm{S}+\mathrm{FEV}=$ antibiótico + suporte + fluido endovenoso; SUPORTE = suporte + fluido endovenoso; $\mathrm{ATB}+\mathrm{S}+\mathrm{SO}=$ antibiótico + suporte + solução oral.

\section{DISCUSSÃO}

Com base nas avaliações sanguíneas, observouse que os animais do grupo SUPORTE apresentaram níveis de linfócitos superiores ao valor fisiológico $(3.008$ a $7.744 / \mu \mathrm{L})$ para bezerros até 30 dias (Peixoto et al., 2002). Esse grupo, diferentemente dos demais, não recebeu aplicação de enrofloxacina (quinolona). A rápida ação desse fármaco é responsável por inibir a síntese de ácidos nucleicos, bloqueando a ação da enzima DNA-girase (topoisomerase II), a qual é responsável pelo acondicionamento e descondicionamento do DNA hiperelicoidal, consequentemente, impedindo a multiplicação e o crescimento dos microrganismos (Rocha et al., 2011; Katzung, 2014). 
No grupo SUPORTE, não houve diminuição da proliferação dos agentes infecciosos, o que fez com que os linfócitos, células responsáveis por realizar o reconhecimento dos antígenos por meio de receptores de membrana específicos para microrganismos invasores, estivessem em maior número, na tentativa de diminuir a resposta infecciosa (Pilla et al., 2012). Os linfócitos agem em estágios mais tardios da inflamação, quando a ação de macrófagos e neutrófilos não é suficiente para deter os microrganismos (Murphy, 2014). Em razão disso, acredita-se que esses animais estivessem enfrentando uma infecção exacerbada e apenas o suporte não foi suficiente para combater a infecção, que refletiu na maior porcentagem de animais com estertoração pulmonar nas $72 \mathrm{~h}$ pósdiagnóstico, em comparação aos animais dos demais grupos. Em relação aos maiores níveis de linfócitos do grupo ATB comparado ao grupo $\mathrm{ATB}+\mathrm{S}+\mathrm{SO}$, sugere-se que a solução oral forneceu equilíbrio hídrico, eletrolítico e, por meio da disponibilidade de glicose, suporte energético, o que possibilitou que os animais tivessem uma resposta à inflamação melhor do que os do grupo ATB.

Já os níveis de neutrófilos segmentados foram maiores no grupo $\mathrm{ATB}+\mathrm{S}+\mathrm{SO}$ em comparação aos demais grupos. Segundo Peixoto et al. (2002), o valor fisiológico de referência de bezerros até 30 dias para neutrófilos segmentados é de 2.790 a 7.190 quantidade/uL e, pelo gráfico, pode-se observar que os animais do grupo $\mathrm{ATB}+\mathrm{S}+\mathrm{SO}$ tiveram um forte recrutamento dessas células para o sangue, até $72 \mathrm{~h}$ após o diagnóstico. Quando ocorre uma infecção, o complexo imune ativa a cascata inflamatória, e os primeiros leucócitos recrutados para o local da infecção são os neutrófilos (Fratric et al., 2012). Esse recrutamento é feito rapidamente pelas citocinas e/ou quimiocinas pró-inflamatórias liberadas pelos macrófagos no momento do reconhecimento do patógeno, estabelecendo-se o estado de inflamação (Pilla et al., 2012; Murphy, 2014). Assim, o uso de antibiótico associado com a solução oral eletrolítica que os animais do grupo $\mathrm{ATB}+\mathrm{S}+\mathrm{SO}$ receberam facilitou o combate contra a infecção, com um grande número de neutrófilos recrutados, os quais foram eficientes para combater a infecção.
Em relação aos eosinófilos, todos os grupos apresentaram níveis dentro dos valores fisiológicos para bezerros até 30 dias de vida, que variam de zero a 415 quantidade /uL (Peixoto et al., 2002). Porém, mesmo estando dentro do padrão para a espécie, os níveis de eosinófilos no grupo $\mathrm{ATB}+\mathrm{S}+\mathrm{SO}$ foram menores em comparação aos demais grupos. Os eosinófilos contribuem para as reações inflamatórias alérgicas e são importantes principalmente na defesa contra parasitas, os quais são muito grandes para serem ingeridos pelos macrófagos ou neutrófilos (Murphy, 2014).

$\mathrm{Na}$ avaliação da contagem de hemácias, o grupo CONTROLE apresentou valores maiores que os grupos ATB, ATB+S+FEV e SUPORTE, não diferindo do grupo $\mathrm{ATB}+\mathrm{S}+\mathrm{SO}$. Além disso, o grupo $\mathrm{ATB}+\mathrm{S}+\mathrm{SO}$ teve maior número de hemácias que o grupo ATB+S+FEV. Uma menor contagem de hemácias nesses grupos (ATB, ATB+S+FEV e SUPORTE) pode ser atribuída à maior gravidade do processo infeccioso, em que ocorre uma disputa pelo ferro entre o organismo e os patógenos (Drakesmith e Prentice, 2012). A hepcidina é um peptídeo produzido principalmente pelos hepatócitos e tem importante função na homeostase do ferro; sua elevação tem sido associada à ocorrência de infecção (Antunes e Canziani, 2016). O ferro é indispensável para a formação da hemoglobina (Baker e Morgan, 1994) e quanto menor a sua concentração sanguínea, menor será a contagem de hemácias. Entretanto, em todos os grupos, os animais se mantiveram com valores dentro do fisiológico $\left(6,3\right.$ a 8,9 x $10^{6} \mu \mathrm{L}$ ) (Peixoto et al., 2002).

Os marcadores bioquímicos analisados não apresentaram diferença quanto aos níveis de PPT, ALB, fibrinogênio, globulinas e à relação A:G. O grupo SUPORTE tendeu a apresentar concentração maior de fibrinogênio em comparação aos grupos $\mathrm{ATB}+\mathrm{S}+\mathrm{SO}$, CONTROLE e ATB+S+FEV. O fibrinogênio é considerado uma proteína plasmática importante na inflamação e, por ser precursor da fibrina, tem fundamental função na coagulação, porém, à hemoconcentração, produz um aumento relativo das proteínas totais e não apenas do fibrinogênio (Jain, 1993). A relação proteína plasmática total/fibrinogênio (PPT/F) é usada para distinguir hiperfibrinogenemia causada por doença ou associada à desidratação (Radostits et al., 2002), 
sendo valores de 10 a 15 considerados como concentração de fibrinogênio relacionada com processos inflamatórios, e superiores a 15 considerados normais (Montagner et al., 2014). A relação PPT:F apresentou diferenças entre os grupos: o grupo $\mathrm{ATB}+\mathrm{S}+\mathrm{SO}$ teve níveis maiores que os grupos ATB e SUPORTE, não diferindo dos demais grupos. Além disso, apenas os grupos CONTROLE e ATB+S+SO apresentaram uma relação PPT:F acima de 15.

Em relação ao aumento da FC observado no grupo $\mathrm{ATB}+\mathrm{S}+\mathrm{SO}$ em relação aos grupos $\mathrm{ATB}$, $\mathrm{ATB}+\mathrm{S}+\mathrm{FEV}$ e SUPORTE, acredita-se que, devido à suplementação eletrolítica e energética, ocorreu o aumento de energia disponível na corrente sanguínea, tendo como efeito o aumento desse parâmetro (Martello et al., 2004). Apesar das alterações observadas em relação aos parâmetros hematológicos, os grupos não apresentaram diferença nas avaliações zootécnicas, o que não interferiu no desenvolvimento das bezerras durante o período acompanhado. Os animais estudados apresentaram a doença entre o nascimento e a sexta semana de vida, e as avaliações zootécnicas foram no mesmo período. Os animais que desenvolveram a doença no final do período de avaliação, possivelmente, não chegaram a apresentar atraso no desenvolvimento. Porém, também se salienta que a avaliação diária dos animais permitiu que os tratamentos fossem realizados rapidamente em relação ao início da doença, possibilitando uma recuperação mais rápida e, assim, um desenvolvimento igual aos animais saudáveis.

\section{CONCLUSÃO}

Nas condições deste experimento, o tratamento com antibiótico (enrofloxacina de rápida ação) e suporte (flunixina meglumina e cloridrato de bromexina) e solução oral (suplementação eletrolítica e energética) ofereceu aos animais melhores condições orgânicas para combater a broncopneumonia, em comparação aos demais tratamentos, favorecendo o organismo a desenvolver uma resposta imune efetiva diante da infecção, o que ainda possibilitou a diminuição da população de microrganismos, levando à diminuição do grau de infecção desses animais.

\section{REFERÊNCIAS}

ANTUNES, S.A.; CANZIANI, M.E. Hepcidin: an important iron metabolism regulator in chronic kidney disease. J. Bras. Nefrol., v.38, p.351-355, 2016.

BAKER, E.; MORGAN, H. Iron transport. In: BROCK, J.H.; HALLIDAY, J.W.; PIPPARD, M.J.; POWELL, L.W. Iron metabolism in health and disease. London: Saunders, 1994. p.63-95.

BUAC, M.; MOJSILOVIĆ, S.; MIŠIĆ, D. et al. Circulating immune complexes of calves with bronchopneumonia modulate the function of peripheral blood leukocytes: in vitro evaluation. Res. Vet. Sci., v.106, p.135-42, 2016.

BUCZINSKI, S.; MENARD, J.; TIMSIT, E. Incremental value (Bayesian Framework) of thoracic ultrasonography over thoracic auscultation for diagnosis of bronchopneumonia in preweaned dairy calves. J. Vet. Intern. Med., v.30, p.1396-401, 2016.

CLOSS, G.J.; DECHOW, C. The effect of calfhood pneumonia on heifer survival and subsequent performance. Livest. Sci., v.205, p.59, 2017

DAVIS, J.L.; FOSTER, D.M.; PAPICH, M.G. Pharmacokinetics and tissue distribution of enrofloxacin and its active metabolite ciprofloxacin in calves. J. Vet. Pharmacol. Ther., v.30, p.564-71, 2007.

DRAKESMITH, H.; PRENTICE, A.M. Hepcidin and the iron-infection axis. Science, v.338, p.768-772, 2012.

FRATRIC, N.; GVOZDIĆ, D.; VUKOVIĆ, D. et $a l$. Evidence that calf bronchopneumonia may be accompanied by increased sialylation of circulating immune complexes' IgG. Vet. Immunol. Immunopathol., v.150, p.161-168, 2012.

GONÇALVES, R.C.; KUCHEMBUCK, M.R.G.; CURI, P.R. et al. Diferenciação clínica da broncopneumonia moderada e grave em bezerros. Cienc. Rural, v.31, p.263-269, 2001.

GONÇALVES, R.C.; KUCHEMBUCK, M.R.G.; LOPES, R.S. et al. Diarréia em bezerros: estudo clínico e laboratorial. Vet. Zootec., v.3, p.35-44, 1991. 
GRAHAM, T.W.; BREHER, J.E.; FARVER, T.B. et al. Biological markers of neonatal calf performance: the relationship of insulin-like growth factor-I, zinc, and copper to poor neonatal growth. J. Anim. Sci., v.88, p.25852593, 2010.

HEINS, B.D.; NYDAM, D.V.; WOOLUMS A.R. et al. Comparative efficacy of enrofloxacin and tulathromycin for treatment of preweaning respiratory disease in dairy heifers. J. Dairy Sci., v.97, p.372-382, 2014.

JAIN, N.C. Essentials of veterinary hematology. Philadelphia: Lea \& Febiger, 1993. 417p.

KAARTINEN, L.; SALONEN, M.; ALLI, L. et al. Pharmacokinetics of enrofloxacin after single intravenous, intramuscular and subcutaneous injections in lactating cows. J. Vet. Pharmacol. Ther., v.18, p.357-362, 1995.

KATZUNG, B.G. Farmacologia básica $e$ clínica. 12.ed. Porto Alegre: AMGH, 2014. $1242 \mathrm{p}$.

LANGEL, S.N.; WARK, W.A.; GARST, S.N et al. Effect of feeding whole compared with cellfree colostrum on calf immune status: the neonatal period. J. Dairy Sci., v.98, p.3729-3740, 2015.

MARTELLO, L.S.U.; SAVASTANO JÚNIOR, H.; TITTO, E.A.L. Respostas fisiológicas e produtivas de vacas holandesas em lactação submetidas a diferentes ambientes. Rev. Bras. Zootec., v.33, p.181-1912004.

MILLAR, H.; SIMPSON, J.; STALKER, A. An evaluation of the heat precipitation method for plasma fibrinogen estimation. Journal of clinical pathology, v.24, p.827-830, 1971.

MONTAGNER, P.; WESCHENFELDER, M.M.; PEREIRA, R.A. et al. Eficácia do uso de enrofloxacina de ação rápida no tratamento da diarreia neonatal em bezerros da raça Holandês. Acta Sci. Vet., v.42, p.1-8, 2014.

MURPHY, K. (Ed.). Imuno biologia. 8.ed. Porto Alegre: Artmed, 2014. 888p.

NRC-NATIONAL RESEARCH COUNCIL. Nutrient requirements of dairy cattle.7.ed. Washington: National Academy Press, p.450, 2001.
PEIXOTO, A.P.C.; COSTA, J.N.; KOHAYAGAWA, R.K. et al. Hemograma e metabolismo oxidativo dos neutrófilos de bovinos da raça Holandesa preta e branca Influência dos fatores etários. Rev. Bras. Saúde Prod. Anim., v.3, p.16-20, 2002.

PILLA, R.; SCHWARZ, D.; KÖNIG, S. et al. Microscopic differential cell counting to identify inflammatory reactions in dairy cow quarter milk samples. J. Dairy Sci., v.95, p.4410-4420, 2012.

RADOSTITS, O.M.; GAY, C.C.; BLOOD, C.D. et al. (Eds.). Clínica veterinária - um tratado de doenças dos bovinos, ovinos, suínos, caprinos e equinos. 9.ed. Rio de Janeiro: Guanabara Koogan, 2002. 1737p.

RAISER, A.G. Alterações no equilíbrio hidroeletrolítico. In: RAISER, A.G. Patologia cirúrgica veterinária. Santa Maria: Univresidade Federal de Santa Maria, 2003. p.1-21.

ROCHA, D.P.; PINTO, G.F.; RUGGIERO, R. et al. Coordenação de metais a antibióticos como uma estratégia de combate à resistência bacteriana. Quím. Nova, v.34, p.111-118, 2011.

STANTON, A.L.; KELTON, D.F.; LEBLANC, S.J. et al. The effect of respiratory disease and a preventative antibiotic treatment on growth, survival, age at first calving, and milk production of dairy heifers. J Dairy Sci., v. 95, p. 49504960, 2012.

THRALL, M.A. Hematologia e bioquímica clinica veterinária. 2.ed. São Paulo: Roca, 2007, $688 \mathrm{p}$.

VIRTALA, A.M.K.; MECHOR, G.D.; GRÖHN, Y.T. et al. The Effect of Calfhood Diseases on Growth of Female Dairy Calves During the First 3 Months of Life in New York State. J Dairy Sci., v. 79, p. 1040-1049, 1996.

WALKER, P.G.; CONSTABLE, P.D.; MORIN, D.E. et al. Comparison of hypertonic salinedextran solution and lactated Ringer's solution for resuscitating severely dehydrated calves with diarrhea. J. Am. Vet. Med. Assoc., v. 213, p. 113-21, 1998. 